\title{
Multiagent System Integrating Process and Quality Control in a Factory Producing Laundry Washing Machines
}

\author{
Paulo Leitão, Senior Member, IEEE, Nelson Rodrigues, Claudio Turrin, and Arnaldo Pagani
}

\begin{abstract}
Manufacturing companies are currently forced to reconsider their production processes by adopting more flexible, robust, and adaptive systems, aiming to improve their competitiveness. Multiagent systems (MASs) technology is suitable to address this challenge by providing an alternative way to design these complex systems based on the decentralization of the control functions over distributed entities. This paper describes the installation of a MAS solution in an industrial factory plant producing laundry washing machines. The installed solution focuses on the integration of quality and process control, and contributes to the maximization of the factory profitability facing changing conditions by applying self-adaptation procedures at the local and global levels. The preliminary results show improvements in the production efficiency and product quality, as well as a reduction of the scrap costs.
\end{abstract}

Index Terms-Factory automation, multiagent systems (MASs), reconfigurable manufacturing control, self-adaptation.

\section{INTRODUCTION}

$\mathbf{M}$ ANUFACTURING companies are playing in global markets that demand highly customized products, with high-quality and short-delivery times. Aiming to improve their competitiveness, they are forced to adopt more flexible, robust, adaptive, and responsive systems. This vision for the factories of the future [1] is sustained by the Industrie 4.0 initiative that aims to create intelligent factories by promoting the computerization of traditional industries [2].

Multiagent systems (MASs) [3], [4] are pointed out as suitable to face this challenge by decentralizing the control functions over a network of autonomous and cooperative entities to provide modularity, autonomy, flexibility, robustness, and adaptability. MAS solutions differ from the traditional ones

Manuscript received August 06, 2014; revised December 22, 2014 and March 08, 2015; accepted April 15, 2015. Date of publication May 07, 2015; date of current version July 31, 2015. This work was supported by the EU Commission within the research contract Integration of Process and Quality Control Using Multiagent Technology (GRACE), coordinated by Università Politecnica delle Marche, and having partners SINTEF, AEA srl, Instituto Politécnico de Bragança, Whirlpool Europe srl, and Siemens AG. Paper no. TII-14-0826.

P. Leitão and N. Rodrigues are with the Polytechnic Institute of Bragança, 5301-857 Bragança, Portugal, and also with the Artificial Intelligence and Computer Science Laboratory, 4200-465 Porto, Portugal (e-mail: pleitao@ipb.pt; nrodrigues@ipb.pt).

C. Turrin and A. Pagani are with Whirlpool Europe, Cassinetta di Biandronno I-21024, Italy (e-mail: Claudio_Turrin@whirlpool.com; Arnaldo_Pagani@ whirlpool.com).

Color versions of one or more of the figures in this paper are available online at http://ieeexplore.iee.org.

Digital Object Identifier 10.1109/TII.2015.2431232 due to their inherent capabilities to respond promptly to changing conditions without external intervention [4]. In particular, MAS technology can play a crucial role in the implementation of the cyber-physical systems (CPSs) paradigm, which is a way to realize the Industrie 4.0 initiative. CPSs concern the combination of real-world physical processes and computing systems, forming an extensive network of embedded systems that integrates sensing, actuation, computation, and physical processes [5].

The deployment of MAS in industrial environments is exemplified by the Production 2000+ project that applied agent technology in a production line for producing cylinder heads for diesel engines at the Daimler Chrysler factory plant in Stuttgart, Germany [6]. Rockwell Automation undertook several experiences in the field, namely, developing an agent-based solution that increased the utilization of steel milling processes for the BHP Billiton and a MAS application for the robust and fault tolerant control of the U.S. Navy ship's cooling systems [7]. Another example is the manufacturing execution system (MES) developed by using the holonic manufacturing systems (HMSs) concepts for the American Glass Product (AGP) company, which produces laminated security glass for automotive application [8]. However, in spite of the promising potential, the number of industrial solutions reported in the literature is still reduced, as pointed out by several review surveys, e.g., [9]-[11].

A wider industrial adoption of the MAS technology involves the implementation of a set of actions, as illustrated by [9][11], namely providing solutions running in industry that show the maturity, flexibility and robustness of the technology, and providing a transparent reengineering process, showing that the implementation can be performed smoothly and compliant with the standards in the field. It should be noted that demonstrations in real industrial environments are crucial for increasing the consensus of the MAS technology by industry.

Additionally, and aiming to avoid the scepticism of industry stakeholders, it is necessary to offer agent-based solutions as black boxes. For this purpose, the system complexity should be hidden in such a way that only its functionalities are shown (e.g., in analogy to our cars where complexity is hidden from the driver that only needs to handle their functionalities).

The EU FP7 GRACE (InteGration of pRocess and quAlity Control using multiagEnt technology) project [12], [13] aimed to develop a modular, intelligent, and distributed control system that integrates process and quality control using the 


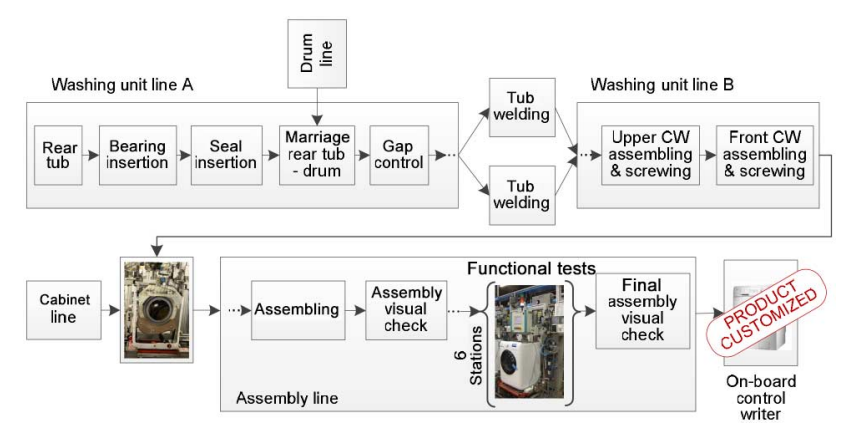

Fig. 1. Layout of the production line.

MAS principles. The objective of this paper is to describe the development and installation of a MAS solution in an industrial factory plant producing laundry washing machines. This application implements real-time monitoring and feedback control loops, which apply dynamic self-adaptation procedures for the online adjustment of process and product variables. The achieved results, in terms of better production efficiency and product quality, contribute for demonstrating the effective applicability of the MAS technology in real industrial environments.

This paper is organized as follows. Section II describes the problem addressed in this work, namely, the production system configuration, objectives, and assumptions. Section III overviews the architectural principles of the designed MAS solution. Section IV presents the details regarding its implementation, installation, and operation in the factory plant. Section V discusses the achieved results by analyzing some qualitative properties and performance indicators. Finally, Section VI rounds up the paper with the conclusion and points out some lessons learned.

\section{Problem Statement}

The problem addressed in this work considers a factory plant owned by Whirlpool and located in Naples, Italy, which produces laundry washing machines. The production layout is composed by several workstations disposed on a sequential order, as illustrated in Fig. 1.

The pallets circulate in the production line through a conveyor system, following a process plan that must be fulfilled to produce the desired washing machine. The process plan contains the information regarding the materials' variables (e.g., type of the rear tub, bearing, or drum) and the operations' parameters (e.g., thickness of the welding process or torque of the bearing insertion process) according to the type of washing machine to be produced.

Each workstation disposed along the production line executes a single operation in the product. The operation can be of the following type: 1) processing (e.g., bearing insertion, tub welding, or pulley screwing); 2) inspection (e.g., gap control, assembly visual check, or functional tests inspection); or 3) manual operation (e.g., electronics and cable assembly). The production execution is performed in a sequential and rigid manner without the dynamic adaptation to unplanned

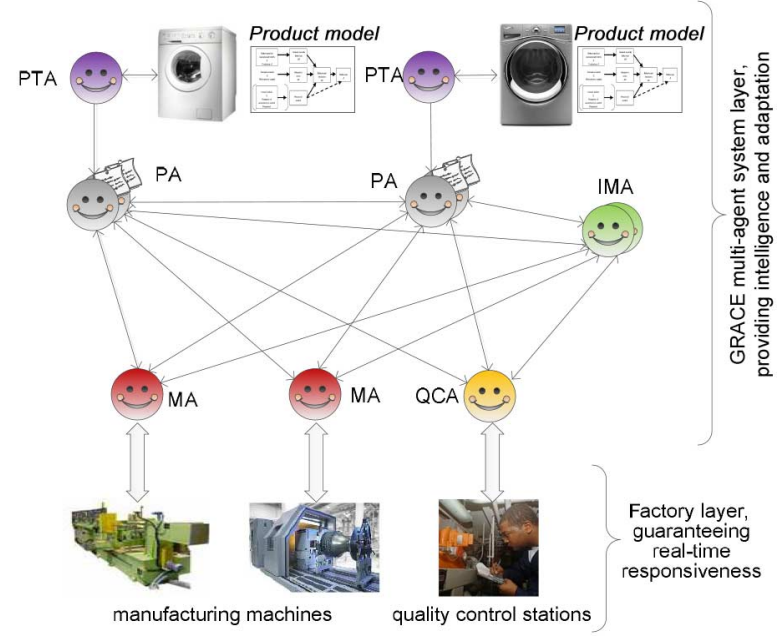

Fig. 2. Agentification of the production line.

changes, which usually occurs in such industrial environments. Unexpected condition changes may occur in the process execution (e.g., slightly deviation of the quality of the tub welding operation) or in materials' characteristics (e.g., deviation of the drum's speed from the expected $3000 \mathrm{rpm}$ to the installed $2950 \mathrm{rpm}$ ). Another example is the functional test area that comprises six stations to execute a set of functional tests to all product instances produced in the production line. Currently, this operation lasts 6 min and comprises a fixed plan, even if some tests may be redundant according to the results already gathered from the previous inspection tests.

This work aims to integrate the process and quality control, establishing feedback control loops to support the on-the-fly adaptation of process and product parameters, to improve the product quality and production efficiency. Examples of such procedures are the adaptation of the operations' parameters, the earlier identification of the quality problems, the customization of inspection tests, and the customization of the final product.

The option to use the MAS technology is motivated by its previously described intrinsic characteristics to face the implementation of self-adaptation procedures addressing a large variety of changes in process parameters. It should be noted that traditional solutions based on centralized and rigid structures exhibit a weak adaptation to condition changes.

An assumption established in this work is to preserve the existing low-level control, based on a network of programmable logic controllers (PLCs) running IEC 61131-3 control programs, which guarantees real-time responsiveness. The MAS solution is placed at higher control level, acting as a backbone for the integration of process and quality control, and providing intelligence and adaptation functionalities [14].

\section{MAS ARCHITECTURE}

The MAS application involves a set of distributed autonomous and cooperative agents representing the components of the production process, as illustrated in Fig. 2.

The agent-based model defines four types of agents according to the particularities of the process to be controlled 
[14]: 1) product type agents (PTAs); 2) product agents (PAs); 3) resource agents (RAs); and 4) independent meta agents (IMAs). PTAs represent the products that can be produced in the factory plant (one agent for each product model). PAs are responsible to manage the orders for the production of product instances (one agent for each laundry washing machine being produced). RAs represent the resources arranged along the production line. Several specializations were considered, namely, machine agents (MAs) associated to processing machines, such as robots or screwing stations, and quality control agents (QCAs) associated to quality control stations. Lastly, IMAs act at strategic level to provide global optimization (they are not mandatory and the system can continue working without them, however compromising its optimization).

\section{A. Modeling the Behavior of Agents}

Several methodologies were introduced in the literature to support the specification and engineering of MAS, such as TROPOS [15] and GAIA [16]. These agent-oriented software development methodologies have their principles on objectoriented programming and are based on the formalization of the organizational level of agents, namely, goals, roles, tasks, and communication patterns. However, these methodologies do not properly capture the environment's dynamic aspects and present difficulties to construct large systems. Unified modeling language (UML) [17] is a modeling language adequate to model object-oriented systems, and agent unified modeling language (AUML) initiative extends the UML to address the requirements of large-scale agent-based applications [18]. However, in spite of its benefits in terms of engineering framework, UML does not efficiently support the formal analysis, simulation, and validation of the system behavior.

In this work, the behavior of each individual agent was modeled by using the Petri nets formalism [19], [20], taking advantage of the well-founded mathematical theory to formally model, simulate, and validate complex event-driven systems exhibiting concurrency, synchronization, resource sharing, and mutual exclusion. The specification of the behavior of the agents is illustrated by modeling the PA using the Petri nets formalism, as shown in Fig. 3.

Briefly, the behavior of the PA comprises several subbehaviors running asynchronously in parallel. The major subbehavior is related to the execution of the process plan (i.e., the path $p 3$ to $p 8$ ). During this subbehavior, the agent decomposes the process plan into $k$ operations and manages their execution taking into consideration the precedences among operations and the current situation of the production line. For this purpose, when the execution of one operation finishes, the next operation to be executed is selected and assigned (represented by the transition 45 ). After the operation's assignment, the agent triggers the supervision of the operation execution, represented by the timed transition $t 6$, which can be expanded into a refined Petri nets model containing the details of this activity, as illustrated in Fig. 4. In particular, the transition $t 6 . t 3$ represents the adaptation of the parameters to execute this operation, e.g., the light condition or the tool wearing calibration, taking into consideration the current production situation and the historical

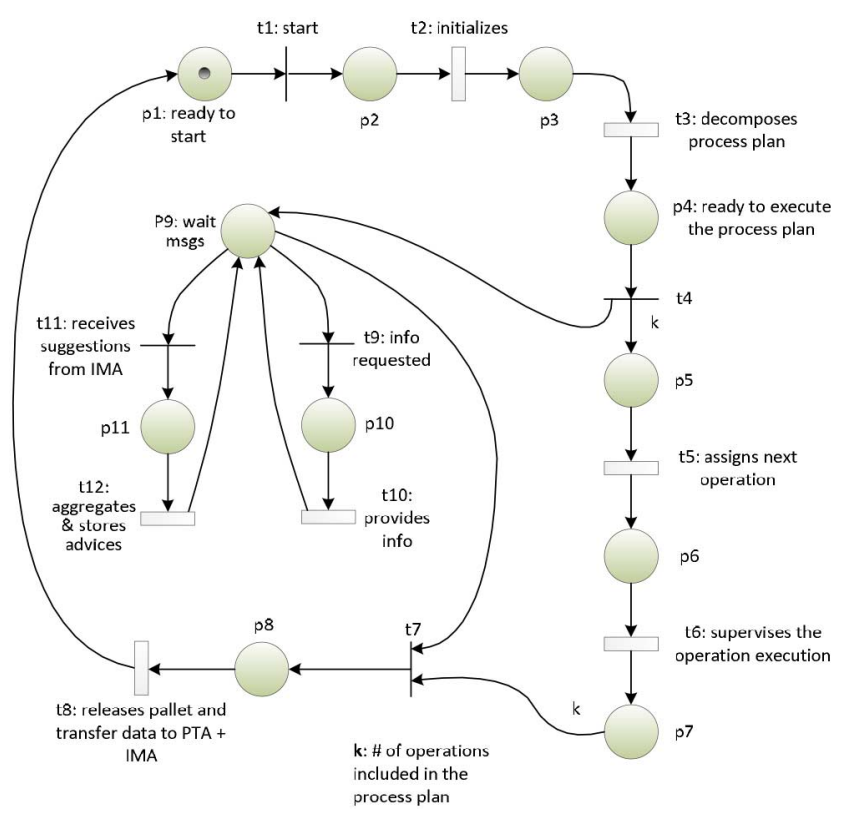

Fig. 3. Petri nets behavioral model of the PA agent.

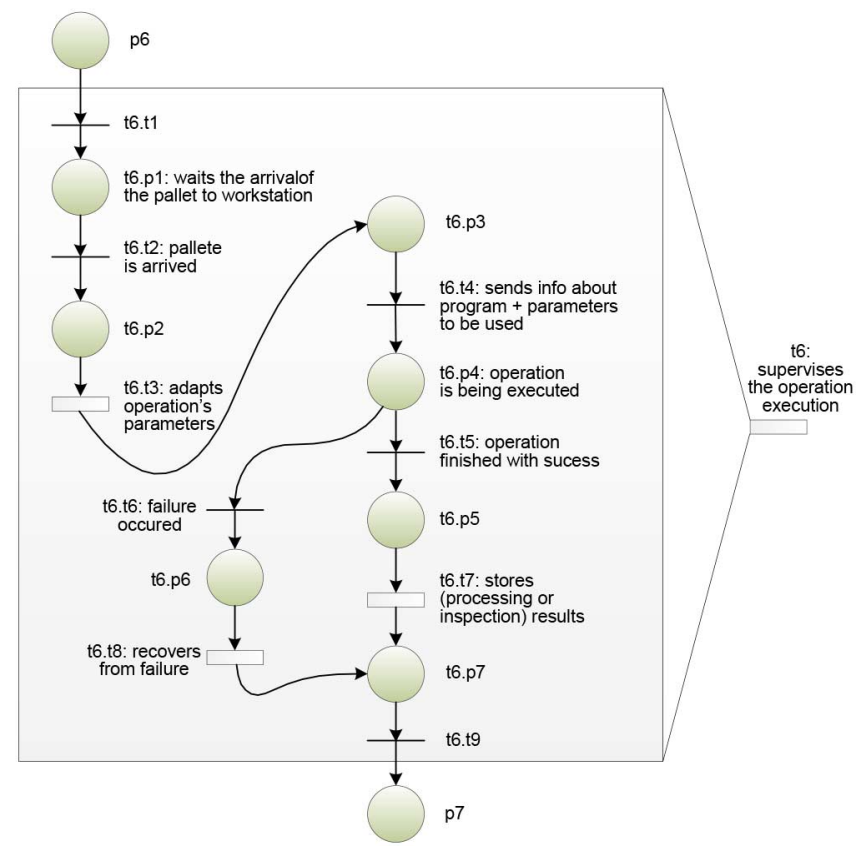

Fig. 4. Petri nets model for the activity "supervises the operation execution."

information of the product execution. It should be noted that this adaptation procedure is only possible due to the complete integration of the feedback information provided by the quality control stations (and associated QCAs).

The conclusion of the process plan is detected when the place $p 7$ is marked with $k$ tokens, representing that all operations were executed. At this moment, the agent releases the pallet and transfers the relevant information to the associated PTA and IMA (represented by the transition $t 8$ ). These agents will use this information for the implementation of posterior global self-adaptation procedures.

Taking advantage of the powerful mathematical foundation associated to the Petri nets formalism, the designed agents' 


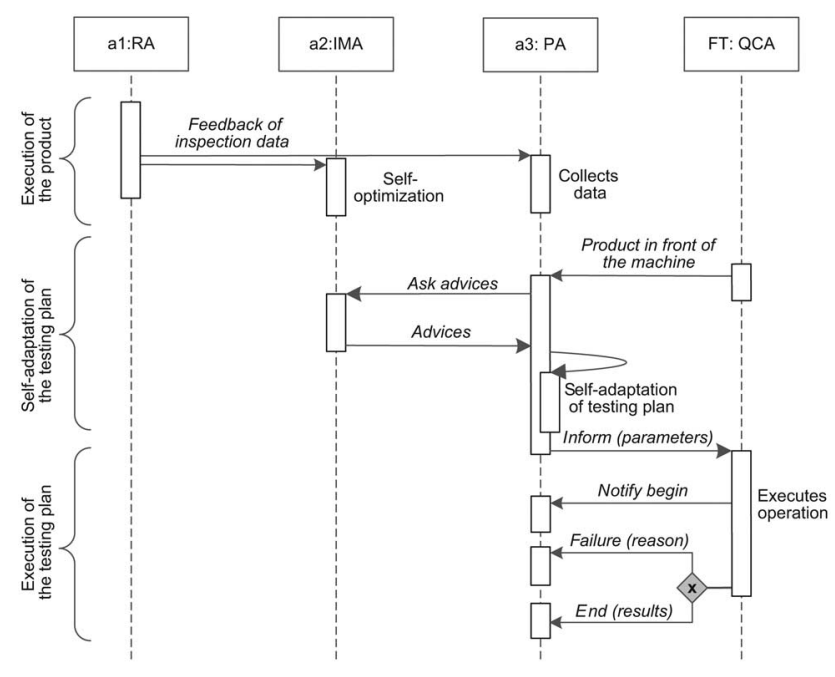

Fig. 5. Interaction pattern for the self-adaptation of the functional tests plan.

models were validated using a qualitative (structural and behavioral) and quantitative (simulation) analysis. This procedure allowed to prove that the models are free of errors and to test if they perform the desired requirements.

\section{B. Modeling the Interaction Supporting Self-Adaptation}

The global behavior of the MAS emerges from the interaction among the agents, each one contributing with its local behavior. For this purpose, several interaction patterns were designed using the AUML sequence diagrams to represent the different coordination forms, composing the complete collaborative multiagent control system.

The interaction patterns aiming the integration of process and quality control for the implementation of self-adaptation procedures are of special relevance in this work. Examples of such designed procedures are the dynamic adaptation of the functional testing plan and the customization of the on-board controller parameters.

PAs implement a procedure to adapt the testing plan to be executed in each washing machine, which requires the interaction among several agents along the production process, as illustrated in Fig. 5.

Briefly, along the production process, the PA collects the feedback data related to the results of the execution of the processing and inspection operations, respectively, from the MA and QCA (see two different implementations of these agents at [21] and [22]). As an example, the MA associated to the bearing insertion station collects data correlating the torque and depth parameters during the execution of the operation. This information is expressed in terms of performance indexes $P j$ reflecting the quality of the execution of each processing operation or inspection test.

When the washing machine reaches the functional tests area, the PA performs a self-adaptation procedure to customize the testing plan for this particular washing machine, taking into consideration the historical production data. For this purpose, the PA calculates the set of variables that defines the sequence of tests in the generic testing rule that is used by the PLC

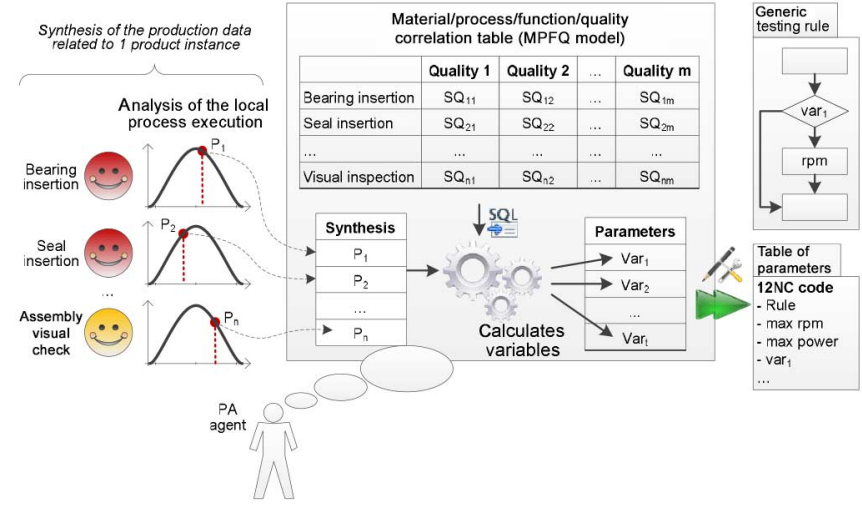

Fig. 6. Customization of the parameters of the functional tests plan.

to control the functional test station. The adaptive function uses the collected $P j$ and correlates them with the materialprocess-function-quality (MPFQ) correlation table, taking in consideration the model of the appliance, as illustrated in Fig. 6 [23]. The PA also uses the advices provided by IMA, based on its wider perspective, for this self-adaptation procedure.

This adaptation procedure allows reducing the inspection time and also executing more efficient tests, particularly by removing unnecessary tests and adjusting others, e.g., the messages showed to the operators.

Another example of a self-adaption procedure is related to the customization of the parameters of the on-board controller installed in each washing machine, performed by PAs at the end of the production line. The on-board controller is responsible to control the operation of the washing machine by executing a default program that is optimized for the optimal production conditions. Since materials and executed processes may be different from those originally designed, PAs correlate the collected data to determine the optimized values for the operation's parameters, e.g., the water flow rate. These parameters are then written in the electrically erasable programmable read only memory (EEPROM) of the on-board controller, allowing to reach a customized washing machine, and consequently improving the product quality.

\section{DEPLOYMENT AND InStALlation IN THE FACTORY PLANT}

This section describes the implementation, installation, and operation of the MAS solution in the factory plant.

\section{A. Implementation of the MAS Application}

The specified MAS application was implemented according to the standards in the field, namely, using the Java Agent Development (JADE) framework that is Foundation for Intelligent Physical Agents (FIPA) compliant. JADE provides a set of functionalities, such as white and yellow pages services [24], which simplifies the development of agent-based applications, as well as a set of auxiliary agents, e.g., agent communication channel (ACC) and agent management system (AMS), which support the life-cycle management of such applications. 


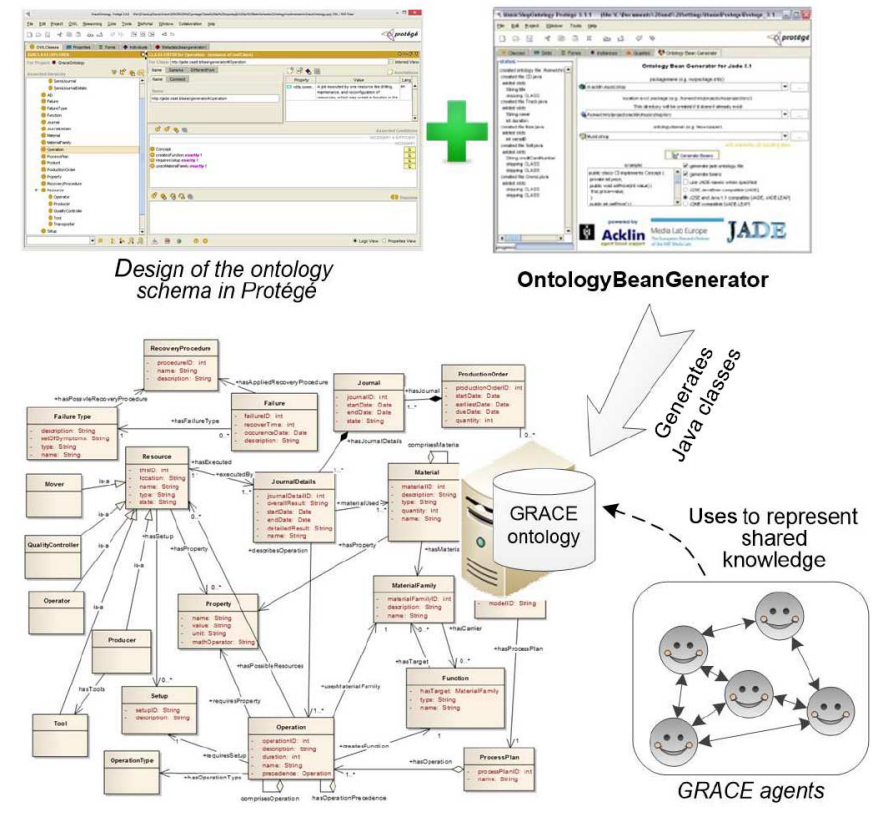

Fig. 7. Integration of the ontology schema in the MAS.

Each type of GRACE agent, namely, PTA, PA, RA, and IMA, is a Java class that extends the agent class provided by the JADE framework according to the specific behavior of the agent (modeled and validated using the Petri nets formalism, as described in Section III). The agents were developed following the JADE's behavior concept [24] that allows the execution of several actions concurrently.

The communication among the distributed agents is asynchronous, i.e., the agent that sends a message continues its execution without the need to wait for the response. To achieve standardized communication, the exchanged messages were encoded by using the FIPA-Agent Communication Language (ACL) [25] and their content formatted according to the FIPASemantic Language (SL0) [26].

The meaning of the messages' content follows the GRACE ontology, which plays a crucial role to enable a common understanding among the agents when they are communicating (at the syntactic and semantic levels). The designed ontology fits on the prerequisites established for the agent-based system integrating process and quality control in production lines, being generic enough within the boundaries of the problem particularities. The ontology schema [27] was designed in Web Ontology Language (OWL) using the Protégé tool [28], and was posteriorly integrated in the MAS application using the OntologyBeanGenerator plug-in, as illustrated in Fig. 7.

An important issue is the integration of the MAS solution in the computational ecosystem that is already running in the production line at different control levels. In this way, the agents are connected with legacy systems, namely, quality control stations, processing stations, MES, and databases. In the case of quality control stations comprising LabView applications, the communication between the agent and the quality control station was performed by using two TCP/IP sockets, as illustrated in Fig. 8.

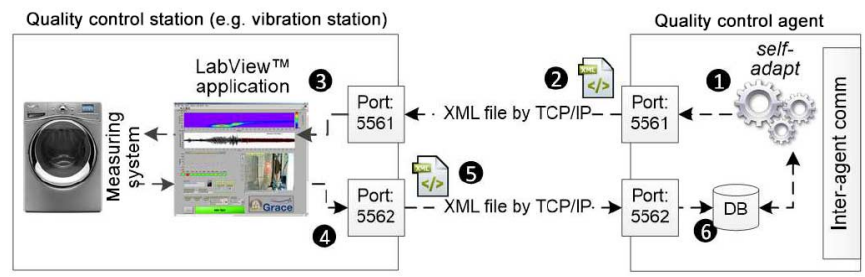

Fig. 8. Interconnection between QCA and quality control station.

A graphical user interface was developed for each type of agent, using the Swing toolkit, providing an easy interface to support the system monitoring and management. Particular attention was devoted to the IMA user interface that provides a global perspective of the production line operation and history upon the data collected from the distributed agents.

\section{B. Installation of the MAS Application}

The agents deployed for the industrial factory plant were as follows.

1) one IMA;

2) eleven QCAs, namely, for the gap control, washing unit inspection, assembly vision check, vibration, final assembly visual check, and six boxes of functional tests workstations;

3) six RAs, namely, for the A-bearing, B-bearing, pulley screwing, screwing upper counterweight, screwing front counterweight, and on-board controller workstations;

4) several PTAs, namely, one agent for each washing machine model.

These agents were distributed by eight computers (mainly, Core 2 Duo, $1.66 \mathrm{GHz}$, and 1 GB RAM) disposed along the production line, as illustrated in Fig. 9. The interconnection among the agents was performed by using TCP/IP over an Ethernet network, as well as the interconnection between the agents and the PLCs located at each workstation.

Agents were customized according to their particularities, namely, the type, list of skills, and set of algorithms, by interpreting an extensible markup language (XML) configuration file during the start-up phase.

PAs are launched on-the-fly by the associated PTAs according to the batch of orders provided by the MES system of the factory plant. In a stable production flow, approximately 400 PAs are running simultaneously.

\section{MAS Solution Running in Practice}

The MAS solution installed in the industrial production system was intensively tested, being possible to analyze its functionality related to monitoring, data analysis, and selfadaptation mechanisms, performed by the individual agents at local and global levels.

As example, RAs are continuously analyzing the performance of their workstations to detect any degradation in their behavior. This allows to anticipate deviations and implement proper self-corrective measures or request external maintenance interventions to mitigate these problems. 


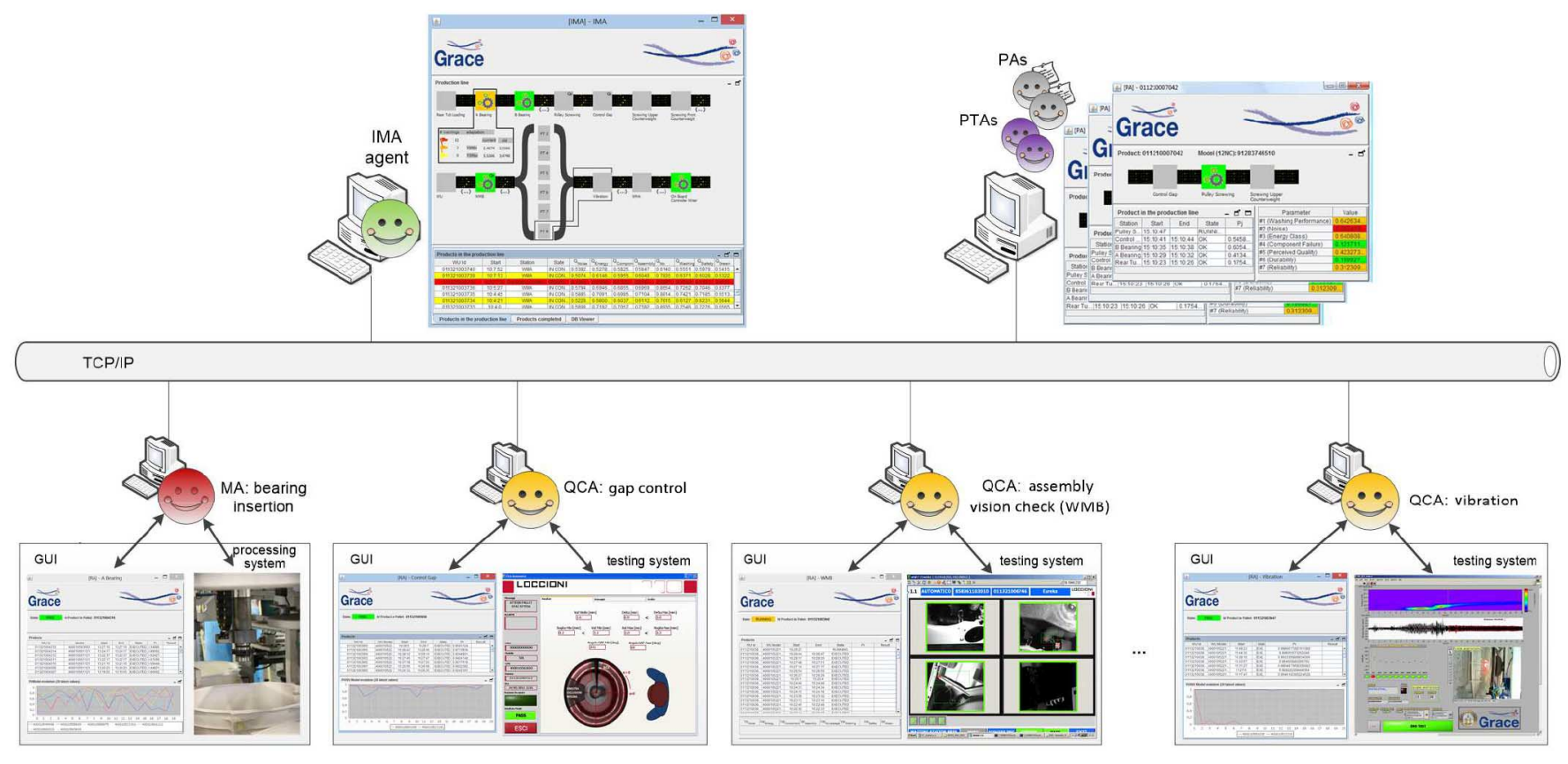

Fig. 9. Distribution of agents in the installed system at the industrial production line.

Using the MPFQ approach, it is possible to correlate the information related to materials, processes, and functions to obtain an estimation of the quality of the washing machine. PAs are continuously analyzing the evolution of these quality indexes, which are related to noise, energy saving, component conformity, assembly conformity, no-leakage, washing performance, safety, and green footprint. The dynamic correlation of this data allows to generate warnings, in any point of the production line, in case the desired quality cannot be achieved anymore, even if the remaining processes will be carried out with a performance of $100 \%$. This mechanism allows identifying earlier possible quality deviations and deciding to remove the washing machine from the production line to save time (i.e., removing earlier a washing machine that will not be quality conformant) and money (i.e., reducing the scraps resulting from producing nonconformant products).

In background, IMAs apply data mining techniques to generate self-optimization guidelines to be proposed to PAs and RAs. As example, IMAs close the feedback loops by using the results of this data analysis to support the dynamic adjustment of up-stream production processes, e.g., the adaptation of the screwing process stations.

Similar approaches based on data-driven techniques can be combined with this agent-based infrastructure; particularly, embedding them in IMA and RAs. As example, a robust data-driven fault detection approach is successfully used for a wind turbine application [29], focusing the data measurement, optimization, and decision-making.

Other data analysis and self-adaptation procedures were implemented and successfully operated at the production line. Particularly, the analysis of the evolution of the quality indexes at station level and the online adaptation of the process plans for each washing machine model, performed by PTAs using the feedback information from PAs.

\section{Discussion OF THE RESUlts}

The operation of the MAS application in the factory plant allowed the achievement of some significant improvements at industrial level, namely, in terms of the following conditions.

1) Increase in the production efficiency: The capability to adapt the process variables based on the feedback loops created by the integration of process and quality control implies an increase in the production efficiency. As an example, the adaptation of the testing plan for each washing machine allowed to reduce, by approximately $20 \%$, the time devoted to perform the functional tests.

2) Reduction of the production down-times: The appliance industry is facing relevant costs for down-time due to the frequent change of models. The self-adaptation of the process parameters permits to reduce the down-time of workstations, by approximately 10\%, when new models are produced. Using this approach, the human intervention is only required for $20 \%$ of new models.

3) Reduction of nonconformities and increase in product quality: The costs of nonquality (e.g., production inefficiency cost and service cost) are also important to be minimized in this type of industry. The introduction of more effective and self-adapted quality control procedures allows to increase the product quality and to reduce the nonconformities of about $1,5 \%$.

4) Increase in the product customization: Each washing machine is customized and its operation optimized due to the adjustment of the parameters of the on-board controller based on the historical production data. This allows achieving better product quality and customer satisfaction.

5) Reduction of the scrap costs: The earlier identification of quality unconformities in the washing machines leads to a reduction of the scraps from $3 \%$ to $5 \%$. Additionally, 
the capability to identify, in an earlier stage, washing machines that never reach the desired quality and to decide to remove them from the production line, contributes to reduce the costs with scraps and also to save production time.

6) Improvement on product energy efficiency: The customization of the parameters of the on-board controller has a significant impact on the sustainability of the domestic appliance use. For example, the calibration of the control flow valve permits to reduce by $50 \%$ the standard deviation in the washing machine water consumption. This reduction implies that more precise washing machine programs are installed, leading to a reduction of $5 \%$ in the water and energy consumption.

These quantitative indicators require a deeper analysis to be performed in a medium-term with the continuous operation of the MAS in the factory plant, particularly analyzing the information collected from longer feedback loops, e.g., the postsales assistance departments.

Beside these quantitative indicators, some qualitative properties were achieved with the installation of the MAS solution, which cannot be reached using the traditional approaches, namely, modularity, flexibility, robustness, on-the-fly reconfiguration, responsiveness, and smooth migration.

In terms of modularity and flexibility, the use of MAS principles simplifies the design and deployment of large-scale CPSs, since they are built upon several building blocks (i.e., the agents). As illustrated in the implemented solution, only four types of agents were developed, namely PTA, PA, RA, and IMA, being the agent-based solution installed in the factory plant populated with as many instances of each one as needed.

The inexistence of a central control node in the developed agent-based solution improves the system robustness, since the system continues operating without perturbation even in case of failure of one agent (which is not possible with traditional centralized and hierarchical structures). As example, a failure in the RA related to the bearing insertion workstation or the IMA does not provoke the stop of the system operation.

The reconfiguration of the computational system can be easily achieved in run-time, since agents can be removed, added, or even modified on-the-fly, i.e., without the need to stop, reprogram, and reinitialize the other agents. This important characteristic, which is not possible in the traditional implementation, was illustrated in several ways, e.g., shutting down an RA associated to the seal insertion station, adding a new RA related to a new box of the functional tests station or changing the algorithm to calculate the performance index embedded in the RA associated to the bearing insertion station.

The integration of process and quality control allowed to implement run-time self-adaptation procedures, embedded in local and global agents, providing fast responsiveness to condition changes in the production process. Examples of such adaptation mechanisms are the customization of the functional tests plan and the on-board controller of each washing machine. Another example is the responsiveness to the change of the product models in the production sequence. As example, during the shift from 8:00 to 16:00 of June 13, four different washing machine models were produced and the system dynamically and on-the-fly understood the introduction of new models in the production line. In fact, the developed self-adaptation and self-optimization strategies permits to cope with complex and variant production processes like the ones involved in the Whirlpool washing machine factory.

Another important characteristic, observed during the operation of the agent-based system installed in the factory plant, is the possibility of smooth migration from the existing technologies or systems to the adoption of new ones. As example, the installed solution considered 12 workstations to be controlled by the agent-based solution, but a slowly and smoothly integration of the remaining workstations along the production line can be performed gradually in time.

At the end, and probably one of the major contributions of this work, is the assessment of the MAS benefits in real industrial environments, by demonstrating its effective applicability in a real industrial factory plant.

\section{CONCLUSION}

In the last decade, MAS is being pointed out as a suitable solution to address the development of complex large-scale systems demanding flexibility, robustness, adaptation, and responsiveness. In spite of the promising perspective, few industrial applications are reported in the literature, which makes difficult the convincement of industry stakeholders on the benefits and maturity of this technology.

This paper describes the implementation and installation of a MAS solution in an industrial factory plant producing laundry washing machines, aiming to integrate process and quality control for the implementation of self-adaptation at local and global levels. The installed MAS application was successfully operated in the industrial plant, allowing to achieve improvements in terms of product quality, production efficiency and adaptability.

Some important lessons were learnt from the experience gathered during the development and installation of the MAS in the industrial production line.

The first lesson is related to the design-deployment path. The use of the Petri nets formalism permitted a rigorous specification, validation and simulation of the agent-based solution during the design phase, which allows the correction and the improvement of control strategies before the practical implementation. During the development process, the balance between the negative impact of stopping the production line for testing the agent-based solution and the advantages of deploying the system in a real environment should be considered. A special attention should be devoted to the technical constraints imposed by industrial production environments, which are very different from those found in simulation and laboratory ones.

Another lesson is related to the use of agent development frameworks. These frameworks offer a plethora of functionalities that facilitates the development, debug, and operation of these systems. However, these frameworks have several weaknesses when addressing industrial environments, namely, requiring:

1) real-time interaction protocols for large-scale systems, ensuring scalability, and latency; 
2) a light ACL protocol to support scalability in large-scale systems (note that the format protocol of FIPA-ACL is too heavy mainly due to the headers in the fields, being required the reduction of the size of data fields);

3) a distributed yellow pages service and discovery mechanism to improve the system robustness [replacing the existence of a centralized node reflecting the directory facilitator (DF) service present in JADE].

The standardization issue is crucial to be addressed, since the implementation of control systems compliant with standards is fundamental for their industrial adoption. For this purpose, agent-based applications should be compliant with the existing industrial standards, e.g., considering the use of OLE for process control-unified architecture (OPC-UA) that enables the transparent, multivendor, interoperability among systems. Another example is the combination of agent-based solutions, placed at higher control level to provide adaptation and intelligence, with the IEC 6113-3 and IEC 61499 standards that are used to ensure the responsiveness to real-time constraints at the lower-control level.

Another issue is related to the integration with legacy systems, which is complex and time consuming since the industrial equipment have usually close and proprietary protocols.

A final remark to note that the successful installation of the agent-based solution in the factory plant contributes for a wider acceptance, by industry stakeholders, of this kind of CPSs.

\section{REFERENCES}

[1] EFFRA. "Factories of the Future PPP: FoF 2020Roadmap," European Factories of the Future Research Association: A Manufuture Initiative, Consultation document, 2010.

[2] T. Böhler, "Industrie 4.0-Smarte Produkte und Fabriken revolutionieren die Industrie," Prod. Mag., May 10, 2012, accessed on Mar. 4, 2014.

[3] J. Ferber, Multi-Agent Systems: An Introduction to Distributed Artificial Intelligence. Reading, MA, USA: Addison-Wesley, 1999.

[4] M. Wooldridge, An Introduction to Multi-Agent Systems. Hoboken, NJ, USA: Wiley, 2002.

[5] C. Lu, R. Rajkumar, and E. Tovar, "Guest editorial special section on cyber-physical systems and cooperating objects," IEEE Trans. Ind. Informat., vol. 8, no. 2, p. 378, May 2012.

[6] K. Schild and S. Bussmann, "Self-organization in manufacturing operations," Commun. ACM, vol. 50, no. 12, pp. 74-79, 2007.

[7] P. Vrba et al., "Rockwell automation's holonic and multiagent control systems compendium," IEEE Trans. Syst. Man Cybern. C, vol. 41, no. 1, pp. 14-30, Jan. 2011.

[8] P. Blanc, I. Demongodin, and P. Castagna, "A holonic approach for manufacturing execution system design: An industrial application," Eng. Appl. Artif. Intell., vol. 21, no. 3, pp. 315-330, 2008.

[9] M. Pechoucek and V. Marik, "Industrial deployment of multi-agent technologies: Review and selected case studies," Auton. Agents Multi-Agent Syst., vol. 17, no. 13, pp. 397-431, 2008.
[10] P. Leitão, V. Marik, and P. Vrba, "Past, present, and future of industrial agent applications," IEEE Trans. Ind. Informat., vol. 9, no. 4, pp. 23602372, Nov. 2013.

[11] V. Marik and D. McFarlane, "Industrial adoption of agent-based technologies," IEEE Intell. Syst., vol. 20, no. 1, pp. 27-35, Jan./Feb. 2005.

[12] GRACE (Integration of Process and Quality Control Using Multiagent Technology) Project [Online]. Available: http://grace-project.org/, accessed on Jun. 14, 2014.

[13] C. Cristalli et al., "Integration of process and quality control using multiagent technology," in Proc 22nd IEEE Int. Symp. Ind. Electron. (ISIE'13), 2013, pp. 1-6.

[14] N. Rodrigues, A. Pereira, and P. Leitão, "Adaptive multi-agent system for a washing machine production line," in Industrial Applications of Holonic and Multi-Agent Systems for Manufacturing, V. Marik, J. Lastra, and P. Skobelev, Eds. New York, NY, USA: Springer, 2013, vol. 8062, pp. 212-223.

[15] P. Bresciani, A. Perini, P. Giorgini, F. Giunchiglia, and J. Mylopoulos, "Tropos: An agent-oriented software development methodology," Auton. Agent. Multi. Agent. Syst., vol. 8, no. 3, pp. 203-236, 2004.

[16] M. Wooldridge, N. Jennings, and D. Kinny, "The Gaia methodology for agent-oriented analysis and design," J. Auton. Agents Multi-Agent Syst., vol. 3, no. 3, pp. 285-312, 2000.

[17] J. Rumbaugh, I. Jacobson, and G. Booch, Unified Modeling Language Reference Manual, 2nd ed. Englewood Cliffs, NJ, USA: Pearson Higher Education, 2004.

[18] B. Bauer and J. Odell, "UML 2.0 and agents: How to build agent-based systems with the new UML standard," J. Eng. Appl. Artif. Intell., vol. 18, no. 2, pp. 141-157, 2005.

[19] T. Murata, "Petri nets: Properties, analysis and applications," Proc. IEEE, vol. 77, no. 4, pp. 541-580, Apr. 1989.

[20] R. Zurawski and M. Zhou, "Petri nets and industrial applications: A tutorial," IEEE Trans. Ind. Electron., vol. 41, no. 6, pp. 567-583, Dec. 1994

[21] S. Serafini, N. Paone, and P. Castellini, "Agent-based station for on-line diagnostics by self-adaptive laser doppler vibrometry," Rev. Sci. Instrum., vol. 84, no. 12, pp. 121703-121713, 2013.

[22] A. Bastari, M. Piersantelli, C. Cristalli, and N. Paone, "Self-adapting test-plans in production line: An application to vision control stations," in Proc. 38th Annu. Conf. IEEE Ind. Electron. Soc. (IECON'12), 2012, pp. 4360-4365.

[23] N. Rodrigues et al., "Adaptation of functional inspection test plan in a production line using a multi-agent system," in Proc. IEEE Int. Symp. Ind. Electron. (ISIE'13), 2013, pp. 1-6.

[24] F. Bellifemine, G. Caire, and D. Greenwood, Developing Multi-Agent Systems with JADE. Hoboken, NJ, USA: Wiley, 2007.

[25] FIPA, "FIPA-ACL agent communication language," Foundation for Intelligent Physical Agents, 2000 [Online]. Available: http://www.fipa. org/repository/aclspecs.html, accessed on Jun. 14, 2014.

[26] FIPA-SL0, "FIPA SL content language specification," Foundation for Intelligent Physical Agents, 2000 [Online]. Available: http://www.fipa. org/specs/fipa00008/XC00008G.pdf, accessed on Jun. 14, 2014.

[27] P. Leitão, N. Rodrigues, C. Turrin, A. Pagani, and P. Petrali, "GRACE ontology integrating process and quality control," in Proc. 38th Аппи. Conf. IEEE Ind. Electron. Soc. (IECON'12), 2012, pp. 4328-4333.

[28] Protégé [Online]. Available: http://protege.stanford.edu/, accessed on Jun. 14, 2014.

[29] S. Yin, G. Wang, and H. R. Karimi, "Data-driven design of robust fault detection system for wind turbines," Mechatronics, vol. 24, no. 4, pp. 298-306, 2014.

Authors' photographs and biographies not available at the time of publication. 